\title{
Clinical Pharmacy Technician Activities and Workforce Requirements at MOH Hospitals during Ten years Mass Gathering Hajj (2006-2015) in Makah and Al-Medina Regions, Saudi Arabia
}

\author{
Yousef Ahmed Alomi*1, Khairat Alhennawi², Nizar Khayayt ${ }^{3}$ \\ ${ }^{1}$ The Past General Manager of General Administration of Pharmaceutical Care and \\ Head, National Clinical pharmacy, and pharmacy practice and Pharmacy $R \& D$ Administration, \\ Ministry of Health, Riyadh, KSA. \\ ${ }^{2}$ Clinical pharmacy staff, General Administration of Pharmaceutical Care, Ministry of Health, Riyadh, KSA. \\ ${ }^{3}$ Medication Safety Officer General Administration of Pharmaceutical care, Makkah Region, \\ Ministry of Health, Riyadh, KSA.
}

\begin{abstract}
Received: 13 August 2017;
Accepted: 28 September 2017

*Correspondence to:

Dr. Yousef Ahmed Alomi,
\end{abstract}

The Past General Manager of General Administration of Pharmaceutical Care Head, National Clinical pharmacy, and pharmacy practice Head, Pharmacy $R \&$ \& Administration Ministry of Health, P.O.BOX 100, Riyadh 11392, Riyadh, Saudi Arabia. Email:yalomi@gmail.com

Copyright: (C) the author(s),publisher and licensee Indian Academy of Pharmacists. This is an openaccess article distributed under the terms of the Creative Commons Attribution Non-Commercial License, which permits unrestricted non-commercial use, distribution, and reproduction in any medium, provided the original work is properly cited.

Publishing Partner : EManuscript [www.emanuscript.in]

\begin{abstract}
Objective: To explore the clinical pharmacy technician activities and workforce requirements at Ministry of Health Hospitals during mass gathering Hajj ten years (2006-2015) at Makah and Al-Medina Regions in Saudi Arabia. Methods: It is a retrospective analysis of ten years (2006-2015) of MOH hospital pharmacies during mass gathering Hajj period (15-30 days). The pharmacy technician should help the clinical pharmacist to provide pharmaceutical to all patients either Pilgrim or not Pilgrim at Makah region. It included Mona holy places hospitals; Arafat holy places hospitals, and Makah city. The workforce requirements calculated based on $\mathrm{MOH}$ workforce standards of hospitals. The clinical activities drive from $\mathrm{MOH}$ critical care services, emergency services, and mortality rate data. The nine clinical pharmacy services characterized by reduction mortality cost saving and mass gathering demand chosen. American College of Clinical Pharmacy (ACCP) model of clinical activities used. Results: The total number of Pilgrims was $(1,952,817-3,161,573)$ with average of $(2,445,208.1)$. The mean number of clinical pharmacy technician needed was (4.28 FTE) per each hospital at Makah. The average number of clinical pharmacy technician needed was (3.2 FTE) per hospital in Al-Medina. The central clinical pharmacy technician activities needs (1 FTE) of clinical pharmacy technician per each hospital in Makah and Al-Madina regions. The patient-centered clinical pharmacy technician services need (2 FTE) of clinical pharmacy technician per each hospital at Makah while (1 FTE) of clinical pharmacy technician per each hospital at Al-Medina region. The administrative, clinical pharmacy technician activities need (1 FTE) of clinical pharmacy technician per each hospital at Makah and Al-Madina regions. Conclusion: The clinical pharmacy technician required to assist the clinical pharmacist activities during mass gathering Hajj period. Both clinical pharmacy technician and clinical pharmacist decrease mortality and morbidity of chronic diseases during mass gathering Hajj time. They improve clinical outcome of illness and prevent the economic burden on healthcare system during mass gathering Hajj periods at $\mathrm{MOH}$ hospitals in Makka and Al-Madina region, Saudi Arabia.
\end{abstract}

Keyword: clinical pharmacy technician, Workload, Workforces, Mass Gathering, Hajj, Makka, Al-Madina, Ministry of Health, Saudi Arabia 


\section{INTRODUCTION}

The hajj is mass Gathering event occurs every year at the minimal area with number 1.5 -2.5 millions of pilgrimage in Makka region or Al-Madina region at a short period. ${ }^{[1]}$ The mass Gathering Hajj is the hugest event in the world. They need the very high amount of medical services and high caliber of healthcare professionals including consultant physician and with expert nurses and clinical pharmacist. The type workforce of health professionals demands to prevent chronic complicated diseases and treatment any critical or emergency situation of any pilgrimage. ${ }^{[2-3]}$ All high qualified personal need another staff to help and support the clinical activities. A clinical pharmacy technician is one of the support the clinical pharmacist with clinical performances. The clinical activities done by pharmacy technician is well known in USA, Canada, and Australia while the author not familiar with any study a bout pharmacy technician in Saudi Arabia or Gulf countries. ${ }^{[4-8]}$ Several studies showed the impact of clinical pharmacy technician through preventing the discrepancy of prescriptions, medication supply increased, more accuracy of taken patient medication history, and increased time for the clinical pharmacist. ${ }^{[9-11]}$ However, It seldom to find any study a bout clinical pharmacy technician workforce during mass gathering like Hajj time over all the world. The goal of the study to explore the clinical pharmacy technician services and workforce requirements during hajj in ten years (2006 -2015) in all places that attended by pilgrims (Makah, holy places, and Al-Medina)

\section{METHODS}

It is a retrospective analysis of ten years (2006-2015) of $\mathrm{MOH}$ pharmacy technician help clinical pharmacists during mass gathering Haji period (15 days) in Makka region and nine years (2006-2014) of $\mathrm{MOH}$ pharmacy technician support clinical pharmacists during mass gathering Hajj period (15-30 days) in Al-Madina region. All data derived from Ministry of Health. Health Statistical Year Books. ${ }^{[12-21]}$ The pharmacy technician helps clinical pharmacist to provide pharmaceutical to all patients either Pilgrim or not Pilgrim at Makah region. It included Mina holy places hospitals; Arafat holy places hospitals, and Makah city. Also, there was extensive literature search review at open date periods with fifty databases included. The search for the term of Hajj and workforce, Hajj and workforce, Hajj and human resources or mass gathering and workforce, mass gathering and workforce, mass gathering and human resources. The search term was in the title and key words. All setting of patient care services hospitals inpatient or ambulatory care or community services included. The search included pharmacy technician help pharmacist and excluded pharmacist and clinical pharmacist. The location of studies included Saudi Arabia as top propriety if not existed Gulf or Middle East countries included, if not found overall counties included. The hospitals included in the study located in Makka city with about ten hospitals and eight Hospitals in Holy places. ${ }^{[2-28]}$ Also, There are about nine hospitals in Al-Medina. ${ }^{[29-32]}$ The workforce requirements calculated based on $\mathrm{MOH}$ workforce standards of pharmacy technician at hospitals. The authors assume $50 \%$ of number help the clinical pharmacist for clinical activities. The clinical activities drive from $\mathrm{MOH}$ critical care services, emergency services, and mortality rate data. The fifteen clinical pharmacy services characterized by reduction mortality cost saving and mass gathering demand chosen. American College of Clinical Pharmacy (ACCP) model of clinical activities used with other literature. ${ }^{[4,7-8,33-35]}$ The clinical pharmacy technician selected activities to support the clinical pharmacist in of fifteen units including drug information, drug utilization evaluation, critical care services, emergency services, cardiology services, infectious disease, pediatrics services, and ambulatory care services. Medication safety, pharmacy research. Also, additional clinical pharmacy services or program impeded or supportive with previous services for instant pharmacy Anticoagulation program, pharmacy pain management program, pharmacy infection control, and total parenteral nutrition clinical pharmacy and therapeutic drug monitoring. The number clinical pharmacy technician selected was twenty-five and divided into the central clinical pharmacy technician activities included Documentation of drug Information Center activities, Prepare medication educational material Check the medication management with compliance during drug utilization evaluation and Participate in clinical pharmacy education to pharmacy technician and nurses, and the public. The patient-centered clinical pharmacy technician activity included screen and interpret of patient laboratory data, the screen of interpreting drug therapy levels, Ensure the medication history and medication available in the pharmacy. Check therapeutic medication plan per guidelines, check patient medication for compliance, Assistant the pharmacist in the transition care system, perform the pharmaceutical calculation. Calculate creatinine clearance, counseling the patient with medication devices techniques, support running ambulatory care clinics (anticoagulation clinic, pain management, diabetics etc.), Identify and Follow up drug related problems, and Assist the pharmacist for therapeutic interchange. The Administrative, clinical pharmacy technician activities included Documentation of Medication Safety reports, Follow up the administration 
related issues of Pharmacy research, Documentation of pharmacy intervention reports, and Documentation of patient counseling. Also, the Another clinical pharmacy technician program included Collection of antibiotic consumption, Follow up the microorganism resistance, Assistant with medication safety survey ISMP, Calculate the cost avoidance of pharmacy activities and Participate in patient drug therapy committees. ${ }^{[4,7-8,33-35]}$ All calculation done used Microsoft Excel version ten.

\section{RESULTS}

There fifteen hospitals in Makka city and holy places while twenty hospitals in Al-Madina region while nine hospitals only mentioned in $\mathrm{MOH}$ statistical book. The majority of clinical pharmacy services at Makka city and holy places where the majority of them provided Drug Information Center 15 (100\%), Antimicrobial stewardship program 15 (100\%), Critical care clinical pharmacy 20 (100\%), and Medication Safety 15 (100\%). While at Al-Madina hospital had Medication Safety Services 20 (100\%), few hospital had Drug Information Center 2 (10\%), and Ambulatory care clinical pharmacy2 (10\%). Both Makka and Al-Madina missed the following clinical pharmacy services including the patient-centered clinical activities with emphasis on Emergency Clinical Pharmacy, Cardiology clinical pharmacy, Pediatrics clinical pharmacy and in addition to Another clinical pharmacy program Pain management program and Anticoagulation programs. The only clinical pharmacy technician activities were Assistant the pharmacist in the transition care system, and Documentation of Medication Safety reports $15(100 \%)$ and $20(100 \%)$ in Makka region and Al-Madina respectively. The most type of clinical pharmacy technician activities missed at all hospitals included The central clinical pharmacy technician activities, The patient-centered clinical pharmacy technician activity, Administrative, clinical pharmacy technician activities, and Another clinical pharmacy technician program $0(0 \%)$ as explored in table 1 and table 2 . The total number of Pilgrims was $(1,952,817-3,161,573)$ with average of $(2,445,208.1)$. In Makah Region; the total number of prescriptions (99,886$257,545)$ with average $(180,120)$. While in Al-Medina Region; the total number of prescriptions $(35,149-207,444)$ with average $(142,080)$. These percentages represent $7.86 \%$ of pilgrims in Makah region and $5.79 \%$ of pilgrims in $\mathrm{Al}-$ Medina respectively. The mean number of clinical pharmacy technician needed was (4.28 FTE) per each hospital at Makah. The average number of clinical pharmacy technician needed was (3.2 FTE) per hospital in Al-Medina. The central clinical pharmacy technician activities needs (1 FTE) of clinical pharmacy technician per each hospital in Makah and
Al-Madina regions. The patient-centered clinical pharmacy technician services need (2 FTE) of clinical pharmacy technician per each hospital at Makah while (1 FTE) of clinical pharmacy technician per each hospital at Al-Medina region. The administrative, clinical pharmacy technician activities need (1 FTE) of clinical pharmacy technician per each hospital at Makah and Al-Madina regions. Other supportive clinical pharmacy technician services need ( 0.28 FTE) of clinical pharmacy technician per each hospital at Makah and (0.2 FTE) Al-Medina regions explored in Table 3 and Table 4.

\section{DISCUSSION}

The clinical pharmacy program started in 2013 at $\mathrm{MOH}$ hospitals and primary care through the general administration of Pharmaceutical Care. ${ }^{[36]}$ The central committee of clinical pharmacy formulated to take of clinical pharmacy system. In addition to more than thirty committee response for each program for an instant but not limited to the following, pharmacy pain management program, pharmacy anticoagulation program, antimicrobial stewardship program, medication safety committee, pediatrics pharmacy program, and pharmacy critical care program. ${ }^{[37]}$ The job description of the clinical pharmacist as general, specialist, or consultant clinical stated. All those programs need the clinical pharmacy technician implemented at $\mathrm{MOH}$ organization by change quality and quantities of a pharmacy technician. All previous program established at regions in Kingdom of Saudi Arabia including the holy cities Makka and Al-Madina. The clinical pharmacy technician can do central clinical pharmacy technician activities, patient specific clinical pharmacy technician activities, or administration clinical pharmacy technician activities. Mass Gathering of Pharmaceutical Care implemented in 2015 and started to apply all previous clinical pharmacy programs including clinical pharmacy technician activities. ${ }^{[38-39]}$ The author through this study tried to evaluate workload and workforces requirements of clinical pharmacy technician during mass Gathering hajj time based on $50 \%$ of $\mathrm{MOH}$ standards pharmacy technician per bed. The remaining time for preparation medication and Pharmaceutical with helping the staff pharmacist. The finding represents the number of pharmacy technician with regular days while the authors suggested the number of clinical pharmacy technician per bed 0.14-0.21 per bed during mass gathering Hajj period. The finding of results can divide through three clinical pharmacy technician above categories. It was new finding the first study done in the worldwide, and it was hard to compare it the other studies. After very extensive literature review an only limited number of studies discussed utilized 
Clinical pharmacy technician workforces at Hospitals during Hajj in Makka Al-Madina

\begin{tabular}{|c|c|c|c|c|c|c|c|c|c|c|c|c|c|c|c|c|c|c|c|c|c|c|c|c|c|c|c|c|c|c|}
\hline & 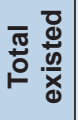 & & ڤ̊ํㅇ & ఏ̊̊̆ & 仓̊ㅇํ & $\begin{array}{l}\stackrel{\circ}{0} \\
\stackrel{0}{0}\end{array}$ & & $\begin{array}{l}\stackrel{\circ}{\circ} \\
\stackrel{0}{0}\end{array}$ & $\begin{array}{l}\stackrel{\circ}{0} \\
0\end{array}$ & $\mid \begin{array}{c}0 \\
\text { ồ } \\
0\end{array}$ & $\begin{array}{l}\stackrel{\circ}{0} \\
0\end{array}$ & 总 & 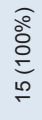 & 总 & 总 & 总 & $\begin{array}{l}\text { ò } \\
\text { ó }\end{array}$ & $\mid$ & $\begin{array}{l}\stackrel{\circ}{\circ} \\
\stackrel{0}{0}\end{array}$ & & 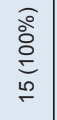 & ठ̊. & 总 & $\begin{array}{l}\text { @̊․ } \\
\stackrel{0}{0}\end{array}$ & & & 总 & $\begin{array}{l}\stackrel{\circ}{\circ} \\
\stackrel{0}{\circ}\end{array}$ & $\mid \begin{array}{c}0 \\
\text { o. } \\
0\end{array}$ & $\stackrel{\circ}{\circ}$ \\
\hline & 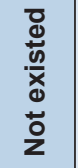 & & 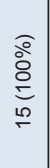 & 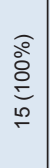 & 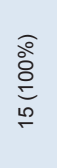 & $\begin{array}{l}\text { ò } \\
\stackrel{0}{\circ} \\
\stackrel{0}{\circ}\end{array}$ & & 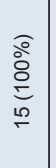 &  & 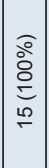 & 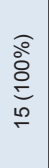 & 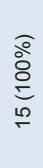 & oे & 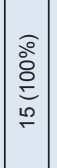 & 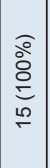 &  & 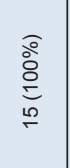 & 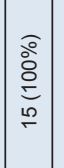 & 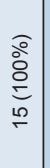 & &  & 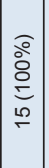 & 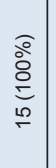 & 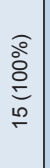 & & 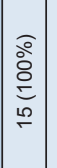 & 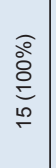 & $\begin{array}{l}\widehat{o} \\
\stackrel{0}{0} \\
\text { co } \\
\underline{c}\end{array}$ & 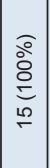 & 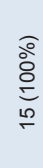 \\
\hline & $\begin{array}{l}8 \\
0 \\
1\end{array}$ & & 0 & 0 & 0 & 0 & & $\circ$ & 0 & 0 & 0 & 0 & 0 & 0 & 0 & 0 & 0 & 0 & 0 & & 0 & 0 & 0 & 0 & & 0 & 0 & 0 & 0 & 0 \\
\hline &  & & 0 & 0 & 0 & 0 & & 0 & 0 & 0 & 0 & 0 & 0 & 0 & 0 & 0 & 0 & 0 & 0 & & 0 & 0 & 0 & 0 & & 0 & 0 & $\circ$ & 0 & 0 \\
\hline & $\begin{array}{l}\text { 웅 } \\
\frac{1}{6} \\
\end{array}$ & & 0 & 0 & 0 & 0 & & 0 & 0 & 0 & 0 & 0 & $\sim$ & 0 & 0 & 0 & 0 & 0 & 0 & & $\sim$ & 0 & 0 & 0 & & 0 & 0 & 0 & 0 & 0 \\
\hline 흠 & $\begin{array}{l}8 \\
\text { ț } \\
\grave{j}\end{array}$ & & 0 & 0 & $\circ$ & 0 & & 0 & 0 & 0 & 0 & 0 & 0 & 0 & 0 & 0 & 0 & 0 & 0 & & 0 & 0 & 0 & 0 & & 0 & 0 & $\circ$ & 0 & 0 \\
\hline 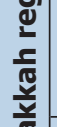 & $\begin{array}{l}\text { ठ্ণ } \\
\text { ஸे } \\
\end{array}$ & & 0 & 0 & 0 & 0 & & 0 & 0 & 0 & 0 & 0 & $m$ & 0 & 0 & 0 & 0 & 0 & 0 & & $\infty$ & 0 & 0 & 0 & & 0 & 0 & $\circ$ & 0 & 0 \\
\hline 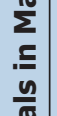 & ণัণ & & 0 & 0 & $\circ$ & 0 & & 0 & 0 & 0 & 0 & 0 & $\sigma$ & 0 & 0 & 0 & 0 & 0 & 0 & & $\sigma$ & 0 & 0 & 0 & & 0 & $\circ$ & $\circ$ & 0 & 0 \\
\hline 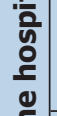 & $\frac{8}{\frac{1}{1}}$ & & 0 & 0 & 0 & 0 & & 0 & 0 & 0 & 0 & 0 & $\theta$ & 0 & 0 & 0 & 0 & 0 & 0 & & $\nabla$ & 0 & 0 & 0 & & 0 & 0 & $\circ$ & 0 & 0 \\
\hline 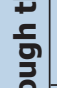 & ధำ & & 0 & 0 & 0 & 0 & & $\circ$ & 0 & 0 & 0 & 0 & $\sim$ & 0 & 0 & 0 & 0 & 0 & 0 & & $\sim$ & 0 & 0 & 0 & & 0 & $\circ$ & 0 & 0 & 0 \\
\hline 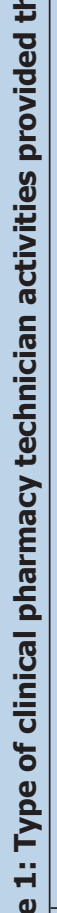 & & 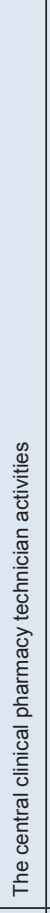 & 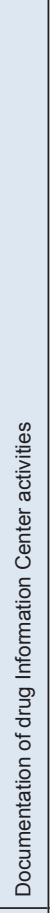 & 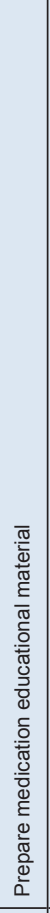 & 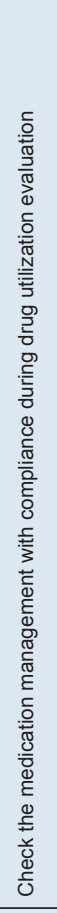 & 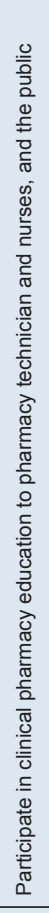 & 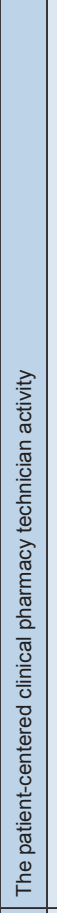 & 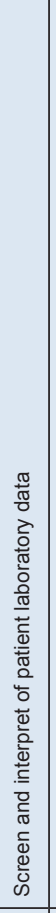 & 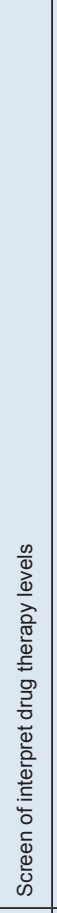 & 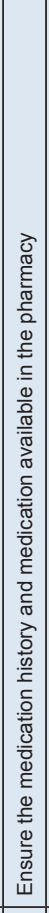 & 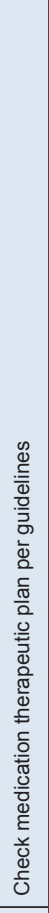 & 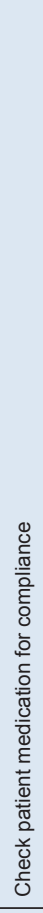 & 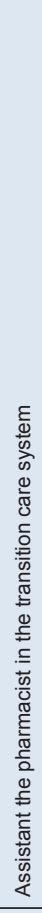 & 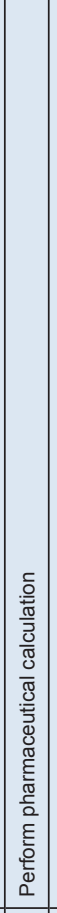 & 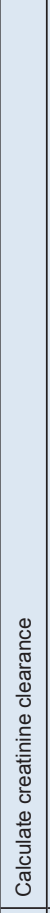 & 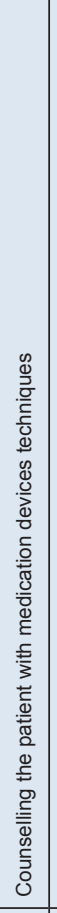 & 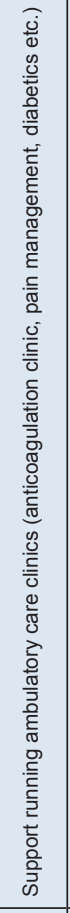 & 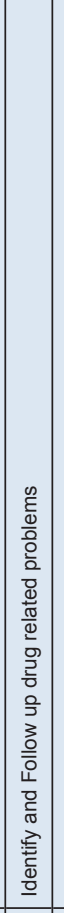 & 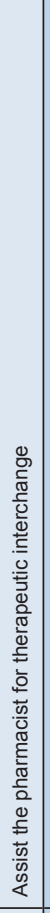 & 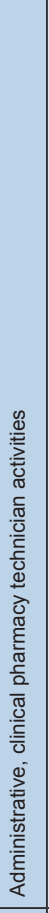 & 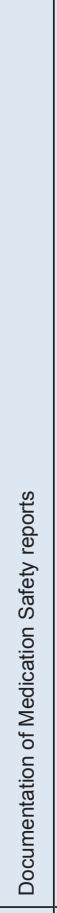 & 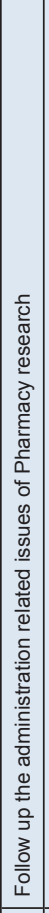 & 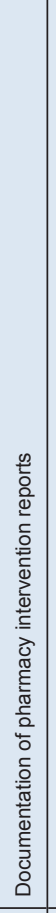 & 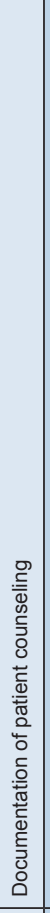 & 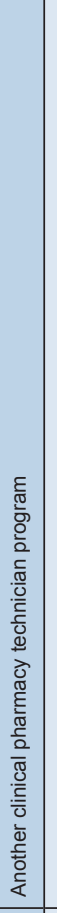 & 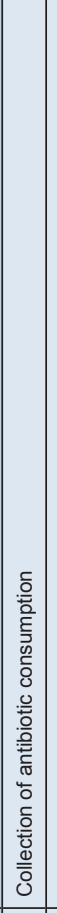 & 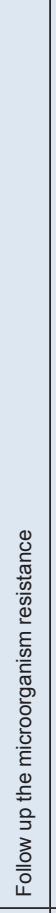 & 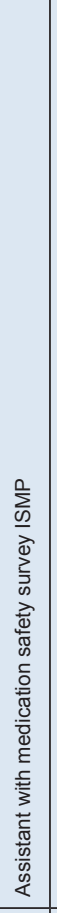 & 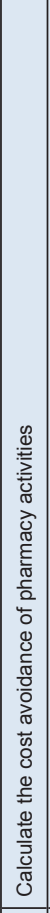 & 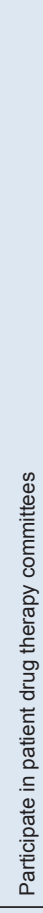 \\
\hline $\begin{array}{l}\overline{0} \\
\vec{\bullet}\end{array}$ & & & - & $N$ & $m$ & $\checkmark$ & & ๑) & 0 & $\wedge$ & $\infty$ & a & 웅 & $F$ & $\cong$ & $\stackrel{m}{=}$ & 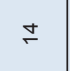 & $\stackrel{2}{\stackrel{2}{\sigma}}$ & $\stackrel{\oplus}{\simeq}$ & & $f$ & $\stackrel{\infty}{\sim}$ & $\stackrel{\rho}{q}$ & 尺 & & $\bar{N}$ & $\approx$ & 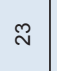 & $\stackrel{d}{ }$ & $\stackrel{\mathscr{N}}{\sim}$ \\
\hline
\end{tabular}




\begin{tabular}{|c|c|c|c|c|c|c|c|c|c|c|c|c|c|c|c|c|c|c|c|c|c|c|c|c|c|c|c|c|}
\hline & 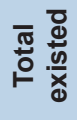 & & 高 & 高 & ฮั & 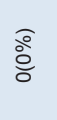 & & 总 & 宮 & 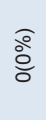 & 总 & 离 & 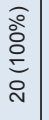 & 高 & ذ̊ & 官 & ฮั & 总 & ठั & 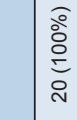 & 高 & 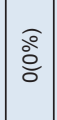 & 咅 & ัे & 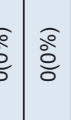 & ذ্. & 高 & ఏ̊ \\
\hline & 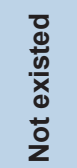 & & 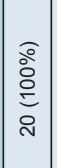 & 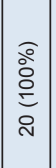 & 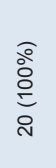 & 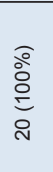 & & 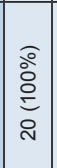 & 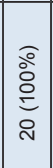 & 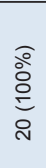 & 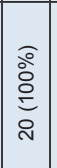 & 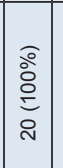 & $\stackrel{\bar{\rho}}{\circ}$ & 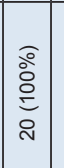 & 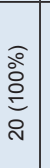 & 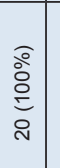 & 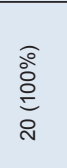 & 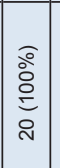 & 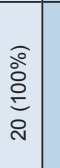 & ฮั & $\mid \begin{array}{l}0 \\
\text { ò } \\
\frac{o}{c} \\
\text { ò }\end{array}$ & 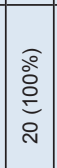 & 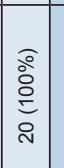 & 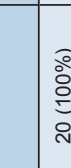 & 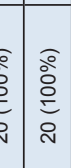 & 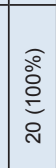 & 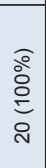 & 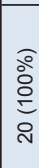 \\
\hline & $\begin{array}{l}\text { ¿े } \\
\wedge\end{array}$ & & 0 & 0 & 0 & 0 & & 0 & 0 & 0 & 0 & 0 & 0 & 0 & 0 & 0 & 0 & 0 & 0 & 0 & 0 & 0 & 0 & $\circ$ & 0 & 0 & 0 & 0 \\
\hline & ¿̊요 & & 0 & 0 & 0 & 0 & & 0 & 0 & 0 & 0 & 0 & - & 0 & 0 & 0 & 0 & 0 & 0 & - & 0 & 0 & 0 & 。 & 0 & 0 & 0 & 0 \\
\hline & 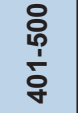 & & 0 & 10 & 0 & 0 & & 0 & 0 & 0 & 0 & 0 & - & 0 & $\circ$ & 0 & 0 & 0 & $\circ$ & - & 0 & 0 & 0 & ○ & 0 & 0 & 0 & 0 \\
\hline 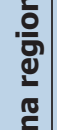 & $\frac{\stackrel{8}{+}}{\grave{j}}$ & & 0 & 0 & 0 & 0 & & 0 & 0 & 0 & 0 & 0 & 0 & 0 & 0 & 0 & 0 & 0 & 0 & 0 & 0 & 0 & 0 & ○ & 0 & 0 & 0 & 0 \\
\hline $\begin{array}{l}\frac{\pi}{\pi} \\
\frac{\pi}{1} \\
\frac{1}{\alpha}\end{array}$ & 莖 & & 0 & 0 & 0 & 0 & & 0 & 0 & 0 & 0 & 0 & $\sim$ & 0 & 0 & 0 & 0 & 0 & 0 & $N$ & 10 & 0 & 0 & $\circ$ & 0 & 0 & 0 & 0 \\
\hline 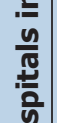 & 啇 & & 0 & 0 & 0 & 0 & & 0 & 0 & 0 & 0 & 0 & $\nabla$ & 0 & 0 & 0 & 0 & 0 & 0 & $\nabla$ & 0 & 0 & 0 & $\circ$ & 0 & 0 & 0 & 0 \\
\hline $\begin{array}{l}\mathbf{T} \\
\mathbf{v} \\
\underline{\underline{y}}\end{array}$ & $\frac{8}{\frac{1}{1}}$ & & 0 & 0 & 0 & 0 & & 0 & 0 & 0 & 0 & 0 & N & 0 & 0 & 0 & 0 & 0 & 0 & $N$ & 10 & 0 & 0 & $\circ$ & 0 & 0 & 0 & 0 \\
\hline 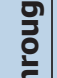 & फ़े & & 0 & 0 & 0 & 0 & & 0 & 0 & 0 & 0 & 0 & 우 & 0 & 0 & 0 & 0 & 0 & 0 & $\stackrel{?}{\circ}$ & 20 & 0 & 0 & $\circ$ & 0 & 0 & 0 & 0 \\
\hline 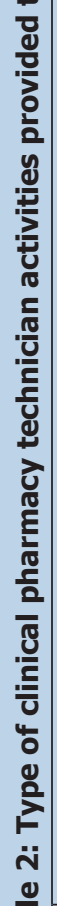 & & 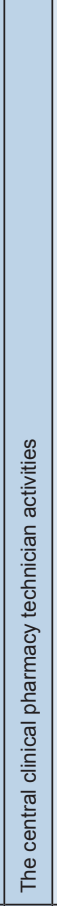 & 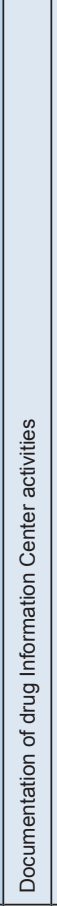 & 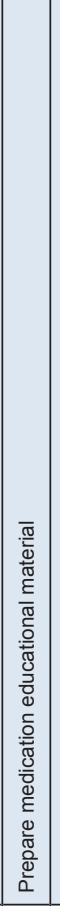 & 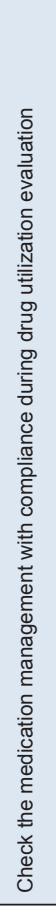 & 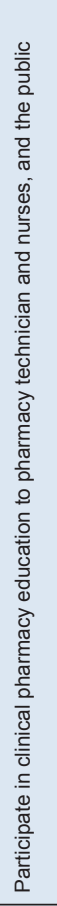 & 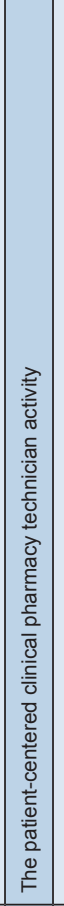 & 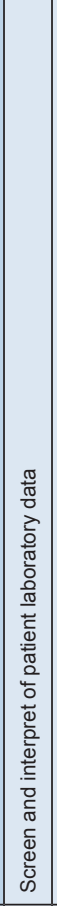 & 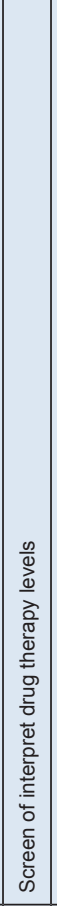 & 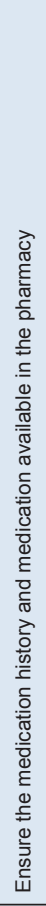 & 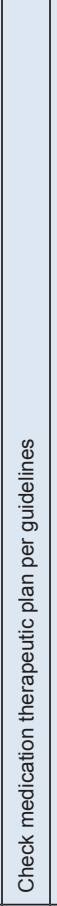 & 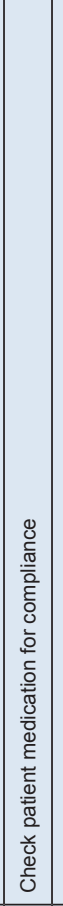 & 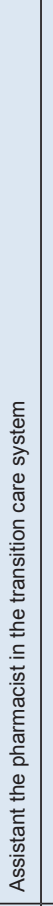 & 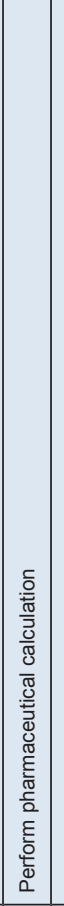 & 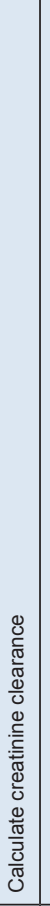 & 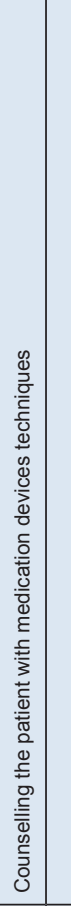 & 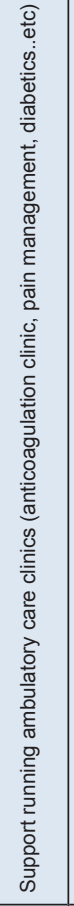 & 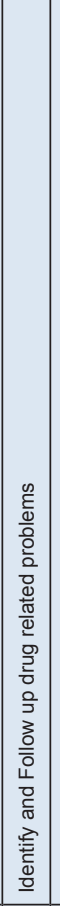 & 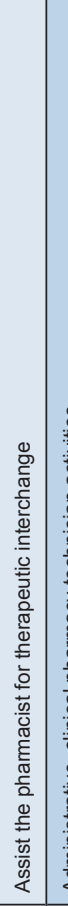 & 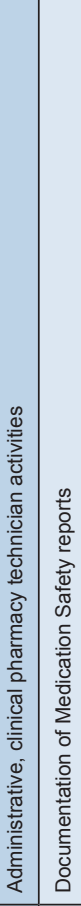 & 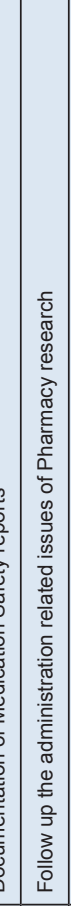 &  & 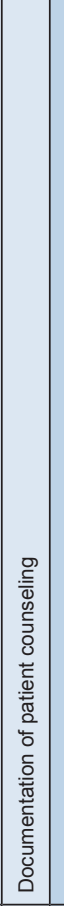 & 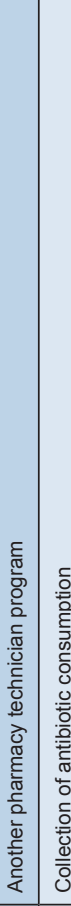 & 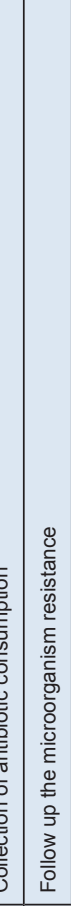 & 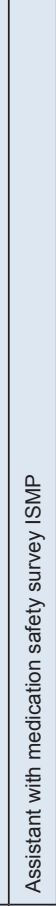 & 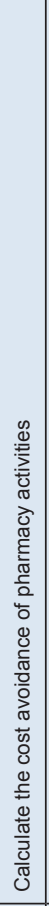 & 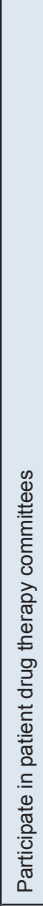 \\
\hline$\pi$ & & & - & w & $m$ & $\sigma$ & & $\infty$ & 0 & r & $\infty$ & o & 웅 & $=$ & $\simeq$ & $\stackrel{m}{\stackrel{2}{2}}$ & $\stackrel{ \pm}{ \pm}$ & $\stackrel{2}{\underline{2}}$ & $\stackrel{\circ}{0}$ & $\therefore$ & $=\stackrel{\infty}{\sim}$ & $\stackrel{9}{\circ}$ & i & $\bar{N}$ & $\bar{v} \mid$ ก & $\stackrel{\infty}{\sim}$ & $\dot{\mathrm{A}}$ & 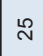 \\
\hline
\end{tabular}



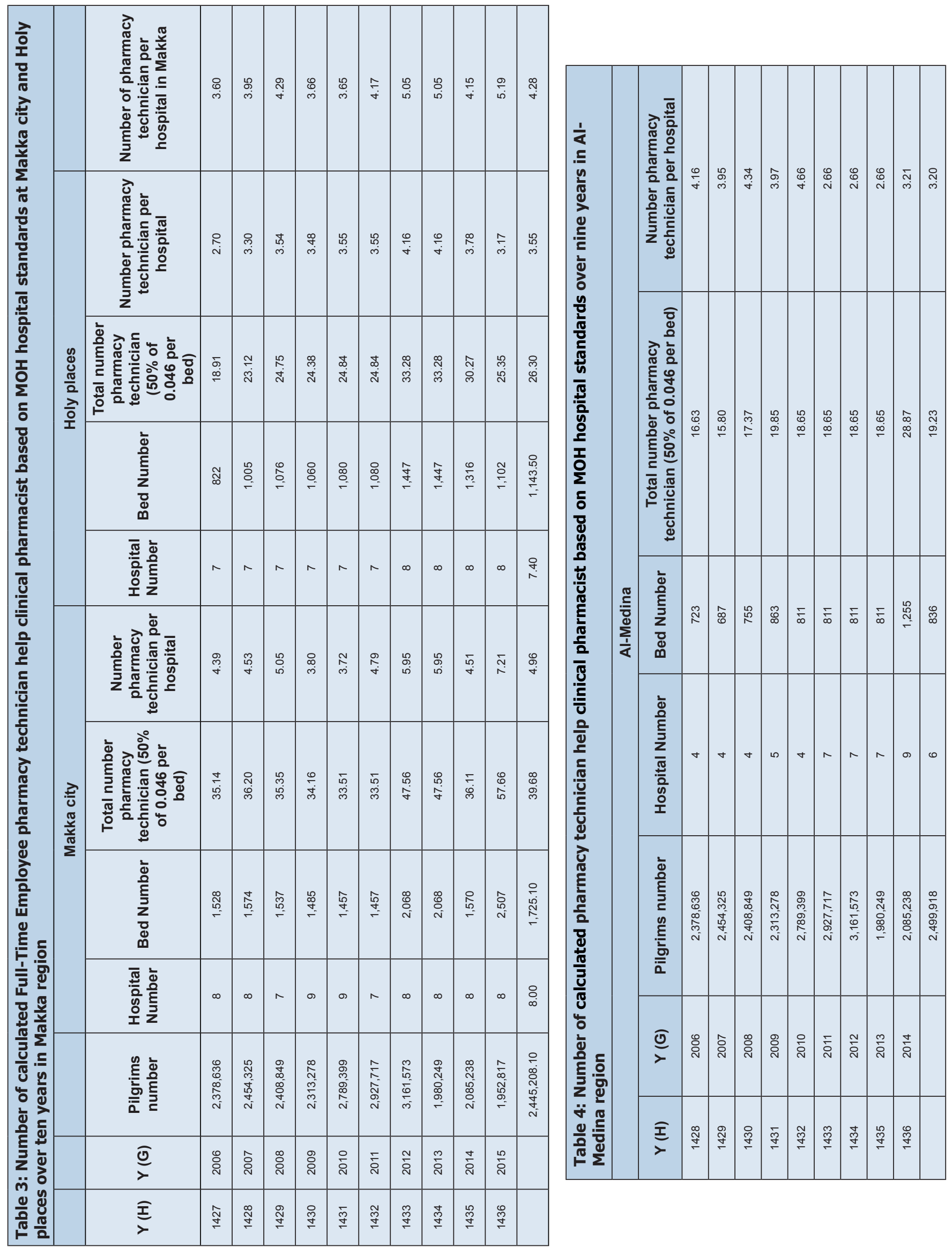
physician and nurses during mass gathering events but not clinical pharmacy technician. ${ }^{[40-42]}$ The study shows the demand, role of the clinical pharmacy technician to help essential clinical pharmacy services and the importance of clinical pharmacy technician especially during mass gathering due to the increasing demand for healthcare services The pharmacist and clinical pharmacists excluded from the study for further investigations in the future.

\section{Limitation}

The study came up with essential and significant results. However, it consisted several limitations. It was not the author's control including the official documentation of clinical pharmacy technician activities missed, missing of published literature locally and in this field, and a cost avoidance of some clinical pharmacy technician activities at both holy cities Makka and Al-Madina.

Conclusion: The clinical pharmacy technician had a significant role in the clinical pharmacy team and services. There is a demand of clinical pharmacy technician during mass gathering Hajj time in Makka or Al-Madina regions. Targeting of setup workforces standards of clinical pharmacy technician during mass gathering Hajj with clinical pharmacy teams may decrease morbidity and mortality, improve patient quality of life, and avoid the economic burden on the healthcare system.

\section{ACKNOWLEDGEMENT}

I want to thank all staff at Health affairs administration and pharmaceutical care administration in Makka and AlMadina regions for their cooperation.

\section{CONFLICT OF INTEREST}

None

\section{ABBREVIATION USED}

KSA: Kingdom of Saudi Arabia, MOH: Ministry of Health,

\section{REFERENCES}

1. Saudi Ministry of Health. Health Statistical Year Book 2016. Available from: http://www.moh.gov.sa/en/ministry/statistics/book/pages/default.aspx

2. Memish ZA. The Hajj: communicable and non-communicable health hazards and current guidance for pilgrims. Euro Surveill. 2010;15(39):19671.

3. Khan NA, Ishag AM, Ahmad MS, El-Sayed FM, Bachal ZA, Abbas TG. Pattern of medical diseases and determinants of prognosis of hospitalization during 2005 Muslim pilgrimage (Haji) in a tertiary care hospital. A prospective cohort study. Saudi Med J. 2006;27(9):1373-80.
4. ASHP. Roles and Responsibilities of Pharmacy Technicians. Am J Heal Pharm. 2016;73:928-30.

5. Mkeresztes J. Role of Pharmacy Technicians in the Development of Clinical Pharmacy. Ann Pharmacother. 2006;40:2015-9.

6. Mabasa VH, Malyuk DL, Tung A, Balen RM, Nicolls TR, Rahiman NL. Using Clinical Pharmacy Support Technicians to Optimize Pharmaceutical Care in the Intensive Care Unit. Can J Hosp Pharm. 2010; 63(1): 41-45.

7. National Association of Pharmacy Regulatory Authorities. Professional Competencies for Canadian Pharmacists at Entry to Practice. 2007. Available from: http://napra.ca/content_files/files/comp_for_cdn_ pharmacists_at_entrytopractice_march2014_b.pdf

8. The Society of Hospital Pharmacists of Australia. Exploring the role of hospital pharmacy technicians and assistants to enhance the delivery of patient centered care . 2016. Available from: http://shpa.org.au

9. Elliott RA, Perera D, Mouchaileh N, Antoni R, Woodward M, Tran T, et al. Impact of an expanded ward pharmacy technician role on service-delivery and workforce outcomes in a subacute aged care service. J Pharm Pract Res. 2014;44(3):95-104.

10. Markovic M, Scott Mathis A, Lee Ghin H, Gardiner M, Fahim G. A Comparison of Medication Histories Obtained by a Pharmacy Technician Versus Nurses in the Emergency Department. P T. 2017; 42(1):41-46.

11. Kraus SK, Sen S, Murphy M, Pontiggia L. Impact of a pharmacy techniciancentered medication reconciliation program on medication discrepancies and implementation of recommendations. Pharm Pract. 2017; 17(2):1-4

12. Saudi Ministry of Health. Health Statistical Year Book 2006. Available from http://www.moh.gov.sa/en/Ministry/Statistics/book/flash/1427/ MOH_Report_1427.html

13. Saudi Ministry of Health. Health Statistical Year Book 2007. Available from http://www.moh.gov.sa/en/Ministry/Statistics/book/flash/1428/ MOH_Report_1428.html

14. Saudi Ministry of Health. Health Statistical Year Book 2008. Available from: http://www.moh.gov.sa/en/Ministry/Statistics/book/flash/1429/ MOH_Report_1429.html

15. Saudi Ministry of Health. Health Statistical Year Book 2009. Available from http://www.moh.gov.sa/en/Ministry/Statistics/book/flash/1430/ MOH_Report_1430.html

16. Saudi Ministry of Health. Health Statistical Year Book 2010. Available from http://www.moh.gov.sa/en/Ministry/Statistics/book/Pages/default.aspx

17. Saudi Ministry of Health. Health Statistical Year Book 2011. Available from http://www.moh.gov.sa/en/Ministry/Statistics/book/Pages/default.aspx

18. Saudi Ministry of Health. Health Statistical Year Book 2012. Available from: http://www.moh.gov.sa/Ministry/Statistics/book/Documents/1433.pdf

19. Saudi Ministry of Health. Health Statistical Year Book 2013. Available from: http://www.moh.gov.sa/en/Ministry/Statistics/book/Pages/default.aspx

20. Saudi Ministry of Health. Health Statistical Year Book 2014. Available from: http://www.moh.gov.sa/en/Ministry/Statistics/book/Documents/StatisticalBook-for-the-Year-1435.pdf

21. Saudi Ministry of Health. Health Statistical Year Book 2015. Available from: http://www.moh.gov.sa/ministry/statistics/book/pages/default.aspx

22. General Directorate of Health Affairs in Makah. Hospitals in Makah Available from: http://mrhb.gov.sa/hospitals/reg/025

23. Al Noor Specialized Hospital. About the Hospital. Available from: http://www. nsh.med.sa/Pages/AboutUs.aspx

24. Ministry of Health. Ibn Sina Hospital Provides Supportive Medical Services to Pilgrims. Available from: http://www.moh.gov.sa/en/Ministry/MediaCenter/ News/Pages/news-2015-09-18-004.aspx

25. Ministry of Health. Ministry News - King Faisal Hospital in Makkah Completes its Preparations for the Pilgrims' Health Care. Available from: http://www. moh.gov.sa/en/Ministry/MediaCenter/News/Pages/News-2012-10-21-003. aspx

26. SA International. King Abdullah Medical City: Mecca, Saudi Arabia. Available from: http://www.sainternational.us/Hospitals/agentType/View/PropertylD/1

27. General Directorate of Health Affairs in Makah. Hospitals in Holy Places. Available from: http://mrhb.gov.sa/hospitals/reg/099

28. Saudi Ministry of Health. Jabal Al Rahmah Hospital Gets Ready to Receive the Hajj Performers on Arafat Day. Available from: http://www.moh.gov.sa/ en/haj/news/pages/news-2013-10-13-013.aspx 
29. Hospital OG. About the Hospital. Available from: http://ohudhospital.com/ cont/s/45

30. Saudi Ministry of Health. Al Anasar Hospital Serviced more than Half Million Pilgrims. Available from: http://www.moh.gov.sa/Directorates/Madinah/ MediaCenter/News/Pages/Page_1435-02-16-007.aspx

31. Al Ansaar General Hospital. Departments \& Services. Ansar Hospital. Available from: http://ansar-eedgroup.com/services/

32. Ministry of Health. MOH Publications - Madinah Health Affairs Directorate Intensifies its Preparations for Serving Pilgrims. Available from: http://www.moh.gov.sa/en/Ministry/MediaCenter/Publications/Pages/ Publications-2013-09-18-001.aspx

33. Bond C, Raehl C, Franke T. Clinical Pharmacy Services, Pharmacist Staffing, and Drug Costs in United States Hospitals. Pharmacotherapy. 1999;19(12):1354-62.

34. Bond C, Raehl C, Franke T. Total Cost of Care, and Length of Stay in United States Pharmacy Services and Staffing. Pharmacotherapy. 2001;21(2):129-41.

35. Bond CA, Raehl CL. Clinical pharmacy services, pharmacy staffing, and hospital mortality rates. Pharmacotherapy. 2007;27(4):481-93.
36. Alomi YA, Alghamdi SJ, Alattyh RA. Strategic Plan of General Administration of Pharmaceutical Care at Ministry of Health in Saudi Arabia 2012-2022. J Pharm Pharm Sci. 2015;1(3):1-8.

37. Alomi YA. National Pharmacy Practice Programs at Ministry of Health in Saudi Arabia. J Pharm Pharm Sci. 2015;1(2):17-8.

38. Alomi Y. National Mass Gathering Pharmaceutical Care Program at $\mathrm{MOH}$ in Saudi Arabia. J Pharm Pract Community Med. 2016;2(23):102-3.

39. Alomi YA. Mass Gathering Pharmaceutical Care Handbook. General Administration of Pharmaceutical Care, Ministry of Health; 2015.

40. Sanders Ab, Criss E, Stecki P, Meislin HWH, Raife J, Allen, et al. An analysis of medical care at mass gatherings. Ann Emerg Med. 1986;15(5):515-9.

41. Smith WP, Wessels V, Naicker D, Leuenberger E, Fuhri P, Wallis LA, et al. Development of a mass-gathering medical resource matrix for a developing world scenario. Prehosp Disaster Med. 2010;25(6):547-52.

42. An Introduction to Mass Gatherings Introduction to Mass Gatherings. 2014; Available from http://www.ceep.ca/publications/Mass_Gatherings.pdf

Cite this article as: Alomi YA, Alhennawi K, Khayayt $\mathrm{N}$. Clinical pharmacy technician activities and workforce requirements at $\mathrm{MOH}$ Hospitals during ten years mass gathering Hajj (2006-2015) in Makah and Al-Medina Regions, Saudi Arabia. J Pharm Pract Community Med. 2017;3(4s):S115-S122. 